\title{
On the Detection of a Non-Cooperative Beam Signal Based on Wireless Sensor Networks
}

\author{
Guofeng Wei $\left(\mathbb{D},{ }^{1}\right.$ Bangning Zhang $\left(\mathbb{D},{ }^{1}\right.$ Guoru Ding $\mathbb{D}^{1},{ }^{1}$ Bing Zhao ${ }^{(D)},{ }^{1}$ Kefeng Guo $(\mathbb{D}){ }^{2}$ \\ and Daoxing Guo ${ }^{1}{ }^{1}$ \\ ${ }^{1}$ College of Communications Engineering, Army Engineering University, Nanjing 210007, China \\ ${ }^{2}$ School of Space Information, Space Engineering University, Beijing 101407, China \\ Correspondence should be addressed to Daoxing Guo; xyzgfg@sina.com
}

Received 8 September 2020; Revised 16 September 2020; Accepted 28 September 2020; Published 19 October 2020

Academic Editor: Xingwang Li

Copyright (C) 2020 Guofeng Wei et al. This is an open access article distributed under the Creative Commons Attribution License, which permits unrestricted use, distribution, and reproduction in any medium, provided the original work is properly cited.

With the extensive research of multiantenna technology, beamforming (BF) will play an important role in the future communication systems due to its high transmission gain and satisfying directivity. If we can detect the non-cooperative beams, it is of great significance in counter reconnaissance, beam tracking, and spectrum sensing of multiantenna transmitters. This paper investigates the wireless sensor networks (WSNs), which is used to detect the unknown non-cooperative beam signal. In order to perceive the presence of beam signals without the prior information, we first derive the detection probability based on the sensors' received signal strength (RSS). Then, based on the strong directivity of the beam signal, we propose an improved " $k$ rank" fusion algorithm by jointly exploiting the energy detection (ED) information and location information of the sensors. Finally, the beam detection performance of different fusion algorithms is compared in simulation, and we find that our proposed algorithm showed better detection probability and lower error probability. The simulation results verify the correctness and effectiveness of the proposed algorithm.

\section{Introduction}

1.1. Background and Motivation. The sixth generation (6G) mobile communication system puts forward some higher requirements for the system capacity, transmission rate, and security [1-4]. Beamforming technology uses spatial gain to meet the needs of increasing system capacity which has become a major trend, focusing limited energy on a specific direction for transmission $[5,6]$. It has been widely studied in recent years for different technologies: millimeter wave (mmWave) [6-8], massive multiple-input multiple-output (MIMO) [9], nonorthogonal multiple access (NOMA) [10, 11], satellite communication [12], vehicular communication [13], device-to-device networks (D2D) [14, 15], etc.

Millimeter wave communication is considered a promising 5G network technology. The frequency of $30-300 \mathrm{GHz}$ is a new area of cellular communication, which provides a larger bandwidth and gains further benefits through beamforming and spatial multiplexing of multielement antenna arrays [7]. Massive MIMO is a new and technically challenging system, and its key feature is to communicate a large number of base station antennas with users through beamforming technology [9]. Through using QAM-64 signals, the massive MIMO millimeter wave transceiver based on beamforming can achieve a stable $5.3 \mathrm{~Gb} / \mathrm{s}$ throughput for a single user in a fast-moving environment [16]. In vehicular communication, beamforming technology is combined with millimeter wave to support the massive automotive sensing [13]. In radar applications, large radio arrays are used for beamforming to obtain enhanced radar performance [17]. The authors also investigated the application of these arrays from an energy and cost-effective perspective to promote new applications $[9,18]$. Besides the above applications, beamforming is often used in the detection, directional communication [19-21], smart surface antenna [22], and covert communication [23].

However, the focus of most research is to combine the beamforming with millimeter wave, massive MIMO, and 
other technologies to increase transmission capacity and reduce interference. Motivated by this fact, if we can detect multiantenna beam signal by WSNs that will bring the following benefits, first, it can better serve the spectrum sensing of secondary users for multiantenna primary users in cognitive radio [24]. Then, by detecting unknown beam signals, we can protect important targets from being discovered. Also, detecting the beam signal and obtaining as much beam information as possible is very significant for multiantenna beam tracking $[15,25]$.

1.2. Related Work. Wireless sensor network uses a large number of miniature sensor computing nodes to conduct real-time monitoring collaboratively through a self-organizing network, perceiving and collecting information about various environments or detection signal [26]. In recent years, with the rapid development of wireless communication technology and electronic device technology, the development and wide application of low-cost, low-power, and multifunctional wireless sensors have become possible. WSNs have been used in battlefield monitoring, environmental perception, search and rescue $[26,27]$, etc.

Beamforming, also known as spatial filtering, is a signal processing technique that shows potentials to significantly raise user throughput, enhance spectral and energy efficiencies, and increase the capacity of mobile networks in the mmWave frequency bands with massive antenna arrays [6, 28]. Many scholars have conducted research and application of beamforming technology. For instance, Zhang [29] introduced the progress and advantages of beamforming technology. Xu et al. [30] proposed a beamforming scheme to enhance the wireless information transmission of terrestrial cellular networks and satellite networks. A fast beam alignment algorithm was investigated for mmWave communications in [31]. Liu [32] applied beamforming technology to cognitive radio cooperative spectrum detection which had better detection performance. The author studied the application of beamforming in the sharing of millimeter wave spectrum between satellites and high-level platform networks and how to better design beams under incomplete channel state information [12]. The author studied the resource allocation design in the cellular mass Internet of Things (IoT) based on NOMA and coordinated the originally harmful cochannel interference in mass access through spatial beamforming $[10,33]$. Yu et al. [34] studied the impact analysis of directional antenna arrays on millimeter wave network coverage.

It can be seen from the above that multiantenna will be more used in future communications, and beamforming technology will play an important role in future communications. However, these research results are all about how to improve the capacity and rate of communication through beamforming technology. At the same time, the authors considered the distributed detection problem, that is, the sensor transmits its local decision through a fully known wireless channel in [35]. The problem of distributed event detection under Byzantine attacks is considered in [36]. The theoretical performance analysis of detection fusion based on conditional dependence and independent local decisionmaking is derived in [37]. The distributed detection of WSNs under multiple receiving antennas fading channels is studied in [38]. However, there is little research on how to perceive a beamforming signal. In accordance with the fact that noncooperative beam signal has the characteristics that the sensors do not work without its beam coverage and lack the priori information. Unlike the previous research on detecting omnidirectional signals [39-41], it is difficult to detect the narrow and directional beam by the single sensor with the previous detection method, so we propose a noncooperative beam signal detection scheme based on WSNs.

1.3. Contributions. The main contributions of this paper are summarized as follows:

(i) We construct an unknown non-cooperative beam signal perception scenario which detects the beam signals lacking the prior knowledge by deploying a large number of sensors to form a network.

(ii) Based on the general ED framework, we derive the detection probability expression under the given false alarm probability and propose a " $k$ rank" fusion algorithm by jointly exploiting the ED information and location information of the sensors.

(iii) To verify the detection performance of the proposed algorithm in the beam scene, we provide some simulation results to demonstrate the effectiveness of the algorithm. In addition, we discuss the impact of different parameters on the detection performance.

The rest of this paper is organized as follows. In Section 2 , the system model is presented and energy detection probability is derived based on the received signal strength of the sensor. In Section 3, we have a brief introduction to the fusion rules. We propose an improved " $k$ rank" algorithm based on distance selection in Section 4. Simulation results and analysis are provided in Section 5. Finally, we conclude the paper in Section 6.

1.4. Notations. For the sake of convenience, we use lowercase and upper-case bold letters represent vectors and matrices, respectively. The key notations used herein are summarized as in Table 1.

\section{System Model}

We consider a system model where WSNs are used to perceive the non-cooperative beam signal, as shown in Figure 1. Suppose an unknown multiantenna transmitter (equipped with $m$ antennas) transmits a plane static beam signal through the beamforming technology. A wireless sensor network is composed of $N$ uniformly distributed sensors where $J$ sensors are within the coverage of the beam signal. The sensor is usually a miniature embedded system that has the ability to perceive physical environment data and process data, but its processing power, storage power, 
TABle 1: Notations throughout this paper.

\begin{tabular}{lc}
\hline Notation & Explanation \\
\hline$P(\cdot)$ & The probability \\
$P(\cdot \mid \cdot)$ & Conditional probability density function \\
$Q(\cdot)$ & Generalized Marcum $Q$ function \\
$\varnothing$ & Empty set \\
$E\{\cdot\}$ & Expectation operator \\
$\exp \{\cdot\}$ & Exponential function \\
$\|\cdot\|$ & Euclidean norm \\
$\mathbb{C}^{m \times n}$ & Complex space of $m \times n$ \\
$\mathscr{C} \cdot \mathcal{N}\left(0, \sigma_{v}^{2}\right)$ & Complex Gaussian distribution with mean 0 and variance $\sigma_{v}^{2}$ \\
\hline
\end{tabular}

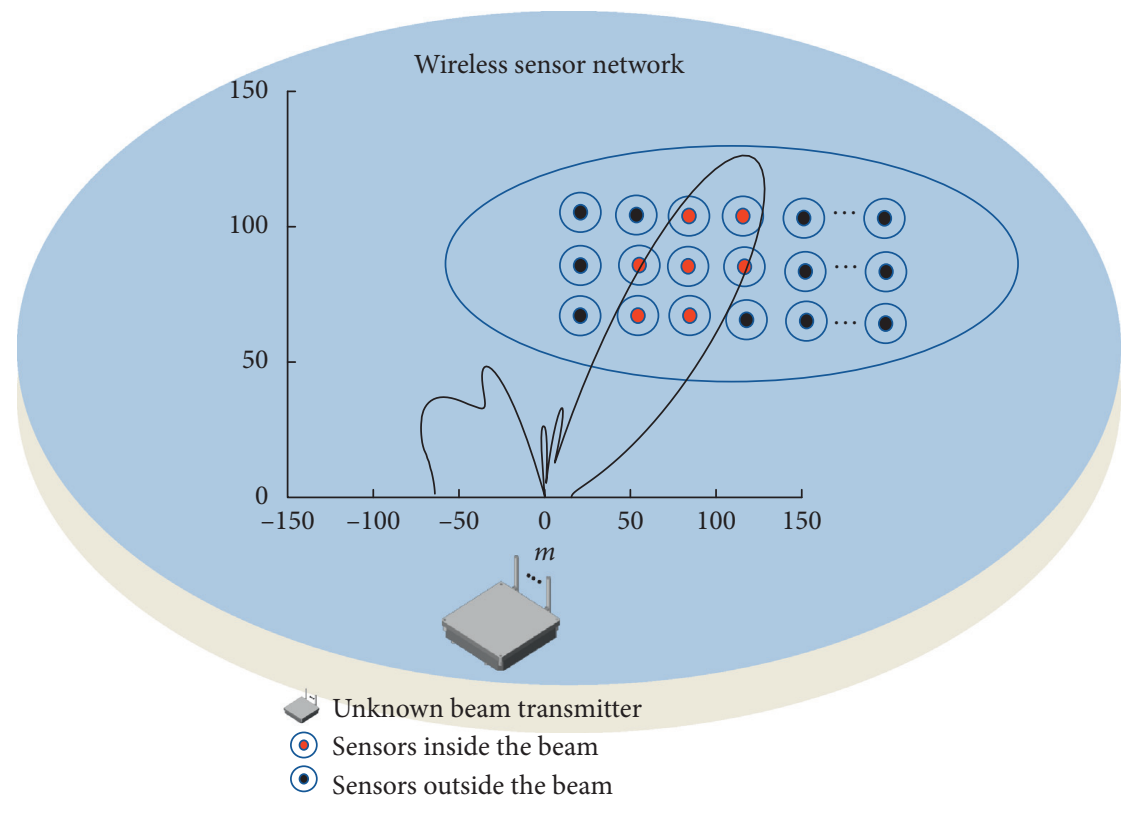

Figure 1: The detection system model of a non-cooperative beam signal based on WSNs.

and communication capabilities are relatively limited. We assume that the fusion center (FC) knows the position information of the sensors by exchanging information.

The received signal strength of $i$-th sensor can be characterized by the following beam signal propagation model:

$$
p_{i}=\mathrm{P}_{t x} g_{i}\left(\frac{4 \pi d_{i} f}{c}\right)^{-\alpha_{n}},
$$

where $g_{i}$ refers to the combined beamforming gain for the link between the $i$-th sensor and unknown multiantenna transmitter, $\mathrm{P}_{t x}$ is the transmit power of unknown multiantenna transmitter, $\alpha_{n} \geq 2$ is power decay coefficient, $c$ is the speed of light, and $d_{i}$ is the distance between the $i$-th sensor and unknown multiantenna transmitter.

According to the signal whether it is in the beam coverage or not, the received signal is

$$
\left\{\begin{array}{l}
y_{\text {inside }}=H_{\text {inside }} x+v, \\
y_{\text {outside }}=H_{\text {outside }} x+v
\end{array}\right.
$$

where $x \in \mathbb{C}^{\mathrm{m} \times 1}$ is the signal emitted by the radiation source and signal variance is $\sigma_{x}^{2}, H_{\text {inside }} \in \mathbb{C}^{\mathrm{m} \times 1}$ and $H_{\text {outside }} \in \mathbb{C}^{\mathrm{m} \times 1}$ are the channel matrix of the beam source ( $m$ antennas) with the sensor in the beam and not in the beam, respectively; the beam signal $x$ is represented as

$$
x=\mathbf{w} u,
$$

where $\mathbf{w} \in \mathbb{C}^{\mathrm{m} \times 1}$ is the beam excursion vector and satisfies $\|\mathbf{w}\|^{2} \leq \mathrm{P}_{t x}$ and $u$ is the coded signal.

A binary hypothetical model at $i$-th sensor is modeled as

$$
y_{i}(n)= \begin{cases}v(n), & H_{0}, \\ H x(n)+v(n), & H_{1},\end{cases}
$$

where $n=1, \ldots, N_{s}, N_{s}$ is the number of samples, $H$ is the channel matrix, and assume that the transmitted signal passes through additive white Gaussian noise (AWGN) channels, so $v(n) \in \mathscr{C} \mathscr{N}\left(0, \sigma_{v}^{2}\right)$.

For non-cooperative beam signals, the energy detection algorithm [42] can be conveniently detected without the prior information. It compares the energy value of the received signal at the sensor in a period with a preset threshold 
to judge whether the target signal exists [43]. When the number of signal samples collected during each sampling period is large enough, according to the central limit theorem, the test statistics is approximately normal distribution. The signal energy value distribution of $i$-th sensor in sampling periods $T$ is expressed as

$$
Y_{i, T}= \begin{cases}\mathcal{N}\left(V_{0}, \frac{\mathrm{v}_{0}^{2}}{N_{\mathrm{s}}}\right), & H_{0}, \\ \mathcal{N}\left(p_{i, T}+V_{0}, \frac{\left(p_{i T}+V_{0}\right)^{2}}{N_{s}}\right), & H_{1},\end{cases}
$$

where $p_{i T}$ is the signal strength received by the $i$-sensor, given by the formula (1), and $V_{0}$ is the noise power.

Then, the detection probability $P_{d, i}$ and the false alarm probability $P_{f, i}$ can be expressed as

$$
\left\{\begin{array}{l}
P_{d, i}=P\left(Y_{i} \geq \gamma_{i} H_{1}\right)=Q\left(\frac{\gamma-\left(p_{i, T}+\sigma_{v}^{2}\right)}{\sqrt{\left(p_{i}, T+\sigma_{v}^{2}\right)^{2} / N_{s}}}\right), \\
P_{f, i}=P\left(Y_{i} \geq \gamma_{i} H_{0}\right)=Q\left(\frac{\gamma-\sigma_{0}^{2}}{\sqrt{\sigma_{0}^{4} / N_{s}}}\right),
\end{array}\right.
$$

where $Q(x)=1 / \sqrt{2 \pi} \int_{x}^{\infty} e^{-\left(y^{2} / 2\right)} \mathrm{d} y$ is the Generalized Marcum $Q$ function.

Under the given constant false alarm probability $P_{f, i}$, the energy detection threshold $\gamma$ can be obtained as

$$
\gamma=\sigma_{v}^{2}\left(1+\sqrt{1 / N_{s}} Q^{-1}\left(P_{f, i}\right)\right) \text {. }
$$

So, the detection probability $P_{d, i}$ can be expressed as

$$
P_{d, i}=P\left(Y_{i} \geq \gamma_{i} H_{1}\right)=Q\left(\frac{\sqrt{1 / N_{s}} Q^{-1}\left(P_{f, i}\right)-p_{i, T}}{\sqrt{\left(p_{i, T}+\sigma_{v}^{2}\right)^{2} / N_{s}}}\right) .
$$

\section{Fusion Rules of Wireless Sensor Networks}

The fusion rules in wireless sensor networks is also called multisensor data fusion technology, which optimizes and merges the sensing data of multiple sensors to obtain more accurate and complete predictions or judgments than single sensor data. There are two main signal processing methods in wireless sensor networks.

The first is that all signals are transmitted to the central processor for processing. There must be no signal delay or signal delay tolerance during the transmission process. Because of the large amount of information to be processed, the bandwidth requirements are relatively high. The second signal processing method is distributed signal processing, that is, the preprocessing of the signal can be completed in the sensor, and only the decision result of the sensor needs to be submitted to the fusion center for fusion. In this way, it requires the least amount of data communication and its requirements for transmission bandwidth are also the lowest. In addition, because the result of FC comes from the optimal synthesis of preliminary judgments of multiple nodes, it has little dependence on a single sensor and the processing cost of the FC is very low. The system has strong anti-interference ability and excellent flexibility in data processing.

Since the data result fusion is a fusion technology for specific decision-making problems, it is generally used to directly obtain the decision-making results of the current problem, so the performance of the fusion algorithm will directly affect the final detection performance. As shown in Figure 2, the sensor submits the quantified judgment result of energy detection to the fusion center and only cares about the signal whether exist, so what is presented here is a binary 0 or 1 . The schematic of fusion center receiving perception results is shown as Figure 3.

Fusion rules can be divided into "AND" fusion, "OR" fusion, and " $k$ rank" fusion.

"AND" fusion: when the judgment result of all sensors is that the signal exists, the final judgment of the fusion center is that the signal exists. As long as one sensor determines that the signal does not exist, the final result of the fusion center is that the signal does not exist. The advantage of the cooperative sensing algorithm based on the "AND" fusion criterion is that the final false alarm probability is very low compared to single sensor perception, but the price is to reduce the detection probability.

"OR" fusion: as long as a sensor judges that the signal exists, it can be considered that the signal exists. Only when all the sensors determine that the signal does not exist, the fusion center can finally determine that the signal does not exist. The cooperative sensing algorithm based on the "OR" fusion criterion has a higher detection probability than single-sensor sensing, because in this method it can be considered that the signal exists as long as there is a sensor to determine the existence of the primary user, and the judgment conditions are relatively loose. But its shortcomings are also obvious, that is, the probability of false alarms will be very high.

" $k$ rank" fusion: the fusion center setting a decision threshold $k$, if there are at least $k$ sensors in the $N$ sensors, the fusion center can determine the signal existence. Otherwise, the fusion center will determine that the signal does not exist. It can be seen that compared to the other two fusion rules, " $k$ rank" fusion has a better application space.

\section{Proposed “ $K$ Rank" Algorithm Based on Distance Selection}

After the fusion center receives the information from the sensors, it will make a fusion decision according to some criteria. " $k$ rank" fusion rule is a fusion decision based on the perception results sent by each sensor, that is, when at least $k$ sensors of the $N$ sensors detect the presence of the signal, the signal is judged to exist. According to the " $k$ rank" fusion rule, the global detection probability $P_{d}$ and global false alarm probability $P_{f}$ of the final judgment result can be expressed as 


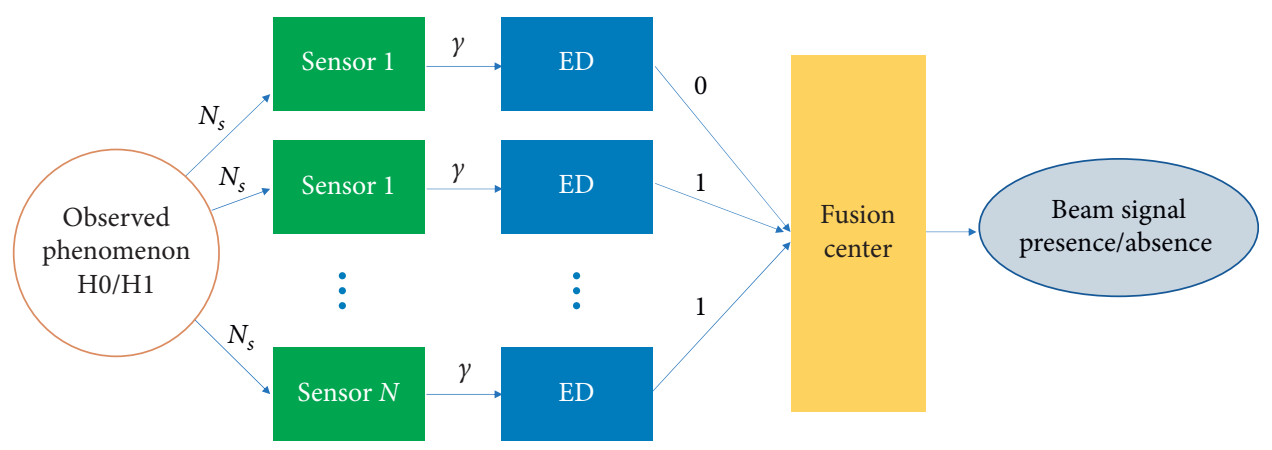

Figure 2: Fusion rules of wireless sensor networks.

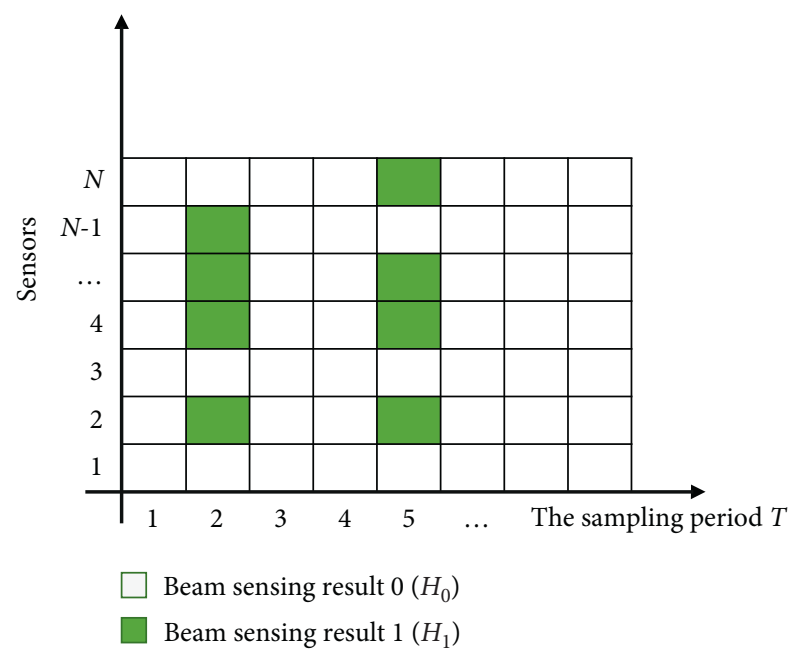

FIGURE 3: Schematic diagram of fusion center receiving perception results.

$$
\left\{\begin{array}{l}
P_{f}=\sum_{k=K}^{N}\left[C_{N}^{k} \times P_{f, i}^{k} \times\left(1-P_{f, i}\right)^{N-k}\right] \\
P_{d}=\sum_{k=K}^{N}\left[C_{N}^{k} \times P_{d, i}^{k} \times\left(1-P_{d, i}\right)^{N-k}\right] .
\end{array}\right.
$$

When $k=1, k=N$, the " $k$ rank" fusion rule is transformed into "OR" fusion rule and "AND" fusion rule. According to the research of M. Schwartz [44], the optimal $k$ of $N$ sensors is approximately $k=1.5 \sqrt{N}$. In addition, there is a half-voting algorithm $k=N / 2$ that is more commonly used.

In order to describe the detection performance of the sensor network better, we introduce the error probability to represent the perceived performance of the system. The error probability mainly consists of two parts: one is the probability which means there is a signal but the result is judged to be absent. The other is the probability which means there is no signal but the result is judged to be a signal. It is expressed specifically as

$$
P_{e}=p\left(H_{0}\right) * P_{d}+p\left(H_{1}\right) * P_{f} .
$$

According to the understanding of the " $k$ rank" algorithm in beam signal perception scenario, we find that only sensors within and around the beam coverage contribute the most to the detection performance of WSNs, while sensors that are not within the beam coverage or far away from the beam sensing area have a limited contribution. If we directly apply the " $k$ rank" fusion algorithm to make detection decisions based on all sensors $(N)$, it will definitely affect the detection performance. Therefore, aiming at the sensing characteristics of the beam signal, a " $k$ rank" fusion algorithm based on distance selection is proposed in order to find the optimal $N_{\text {opt }}$.

We assume that the position information $d_{i, j}$ of each sensor is known by exchanging information with the fusion center, and the distance between any two sensors ( $i$-th sensor and $j$-th sensor) is

$$
d_{i, j}=\sqrt{\left(d_{i}(x)-d_{j}(x)\right)^{2}+\left(d_{i}(y)-d_{j}(y)\right)^{2}} .
$$

We construct a neighbor node library of by finding the nearest neighbor node of $i$-th sensor; $D_{\text {near }}$ is expressed as

$$
D_{\text {near }}(i)=\left\{\text { sensors of } \min \left(d_{i, j}\right)\right\}
$$

We construct a sensing node library of the $i$-th sensor which greater than the detection threshold; $D_{\text {sensing }}$ is expressed as

$$
D_{\text {sensing }}(i)=\left\{\text { sensors of } P\left(Y_{i} \geq \gamma_{i} \mid H_{1}\right)\right\} \text {. }
$$

Because the directionality of the beam signal is very obvious, in order to determine the sensors that detect the signal are concentrated in the beam signal, rather than scattered randomly distributed, we compare the smallest distance between sensors in the sensing node library $D_{\text {sensing }}$ and the smallest distance in the sensor distance library $D_{\text {near }}$. The distance information should satisfy

$$
d_{i j} \min \text { of } D_{\text {sensing }}<=d_{i \text { min }} \text { of } D_{\text {near }} \text {. }
$$

The main steps are summarized as follows: first, calculating the proximity point of each sensor to form the nearest library $D_{\text {near }}$. Second, finding the sensor with the perception result $H_{1}$, and putting the sensor with its neighbor library node together into the sensing library $D_{\text {sensing }}$. Finally, using 


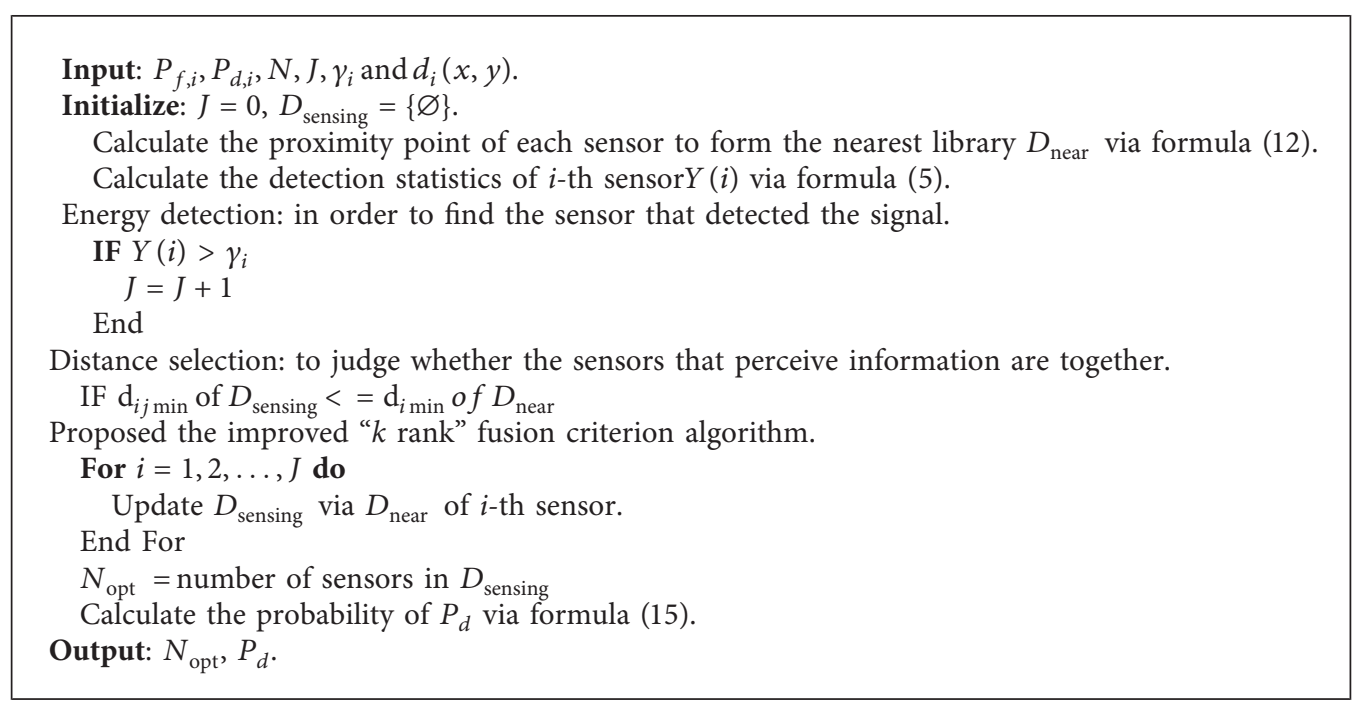

Algorithm 1: $k$ of $N_{\text {opt }}$ fusion algorithm based on distance selection.

$N_{\text {opt }}$ that is the number of sensors in $D_{\text {sensing }}$, instead of the original $N$ for " $k$ rank" fusion. So the $k$ of $N_{\text {opt }}$ fusion is

$$
\left\{\begin{array}{l}
P_{f}=\sum_{k=K}^{N_{\mathrm{opt}}}\left[\mathrm{C}_{N_{\mathrm{opt}}}^{k} \times P_{f, i}^{k} \times\left(1-P_{f, i}\right)^{N_{\mathrm{opt}}-k}\right], \\
P_{d}=\sum_{k=K}^{N_{\mathrm{opt}}}\left[\mathrm{C}_{N_{\mathrm{opt}}^{k}} \times P_{d, i}^{k} \times\left(1-P_{d, i}\right)^{N_{\mathrm{opt}}-k}\right] .
\end{array}\right.
$$

The specific algorithm is shown in Algorithm 1.

\section{Simulation Results}

This section provides simulation results of the beam signal perception to evaluate the detection performance of the proposed algorithm. The simulation parameter [45] settings are shown in Table 2.

Figures 4 and 5 examine the beam pattern information of non-cooperative beam $\left(0^{\circ}-180^{\circ}\right)$. Linear array $(m=8$ antennas) forms a beam signal pointing in $45^{\circ}$ direction, beam excursion vector $w=[0.593+0.805 i, \quad 0.282-0.959 i$, $-0.934+0.357 i, \quad 0.850+0.527 i, \quad 0.850-0.527 i$, $-0.934-0.357 i,-0.734+0.679 i, 0.985+0.173 i]$. Beamforming technology performs signal processing by weighting and combining the signals transmitted by the multiantenna array elements. By designing different weighting factors for the multichannel signals transmitted by multiple antennas and performing signal processing, the effective output of the source signal is improved, which can effectively reduce the interference between users and suppress the influence of noise and obtain the desired signal, thereby improving system performance. It can be seen from Figure 4 that the maximum gain of the beam signal is $18 \mathrm{dBi}$. In Figure 5, it can be seen more intuitively that the main lobe direction of the beam is $45^{\circ}$ and the space distribution between the beam side lobe and the main lobe can be displayed more concretely, which is more conducive to our understanding of beam signals. For convenience, we only show $0-180^{\circ}$ here.
TABLE 2: Simulation parameters.

\begin{tabular}{lc}
\hline Parameters & Value \\
\hline Center frequency & $f_{c}=28 \mathrm{GHz}$ \\
Beam transmit power & $P_{\mathrm{tx}}=30 \mathrm{dBm}$ \\
Number of antennas & $\mathrm{m}=8$ \\
Antenna spacing & $d=\lambda / 2$ \\
Beam pointing angle & $\theta=45^{\circ}$ \\
Beam width & $\mathrm{Bw}=17.8^{\circ}$ \\
Beam maximum gain & $\mathrm{G}=18 \mathrm{dBi}$ \\
Number of sensors & $N=144$ \\
Sensor sensing radius & $r=5 \mathrm{~m}$ \\
\hline
\end{tabular}

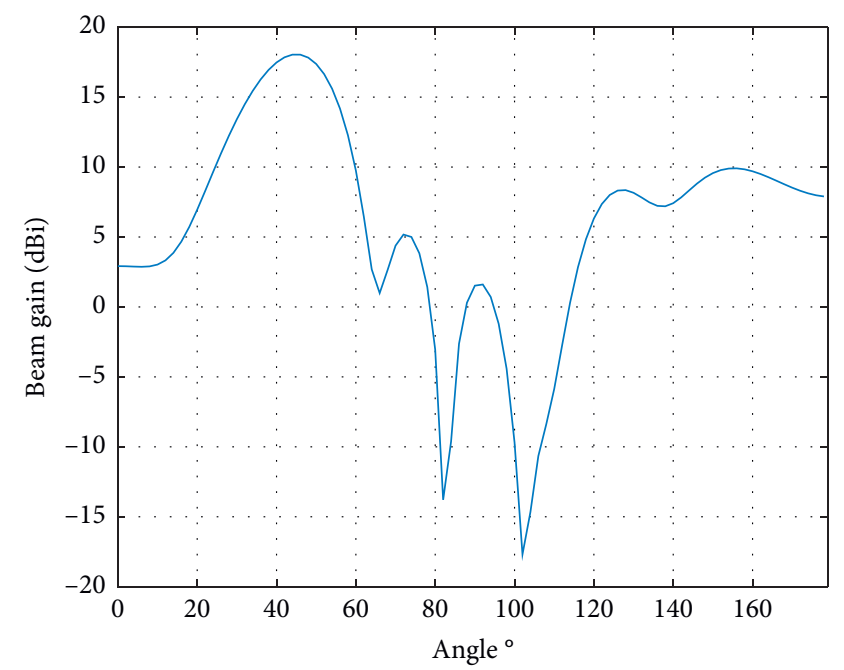

FIGURE 4: Antenna pattern of unknown noncooperative beam.

Since the beam source does not have any priori information for the sensor network, it is difficult to detect the beam signal. With the number of antennas increasing, the beam will become a narrower directional signal, which will bring great challenges to our beam sensing algorithm. 


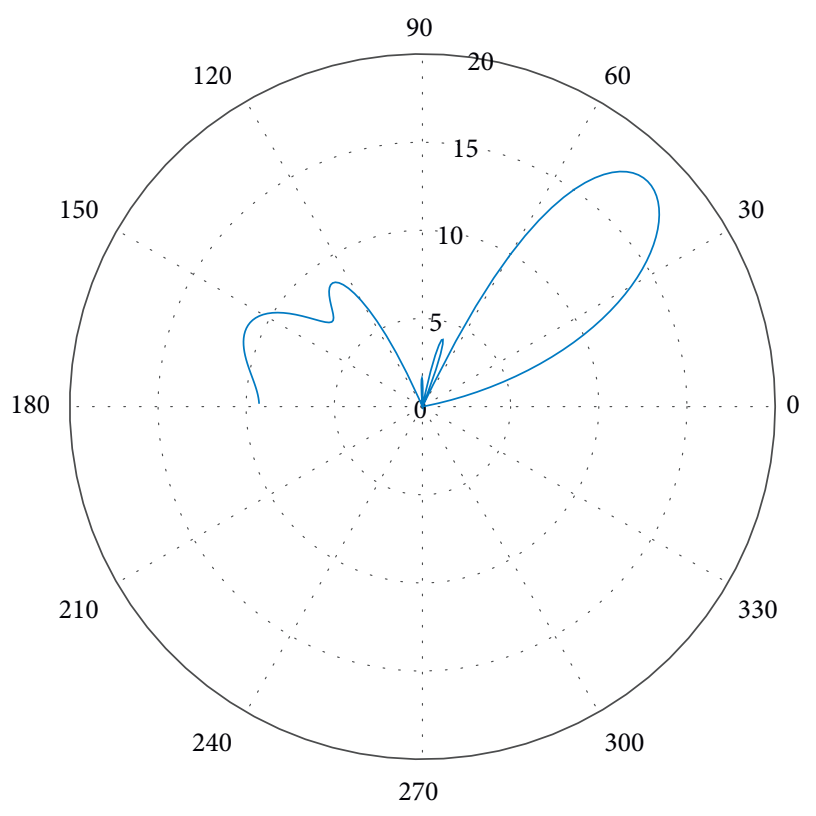

FIGURE 5: Polar antenna pattern of unknown noncooperative beam.

The simulation scene is shown in Figure 6; we deploy a $12 * 12$ uniform sensor network to detect the coverage area, set the sensing range of each sensor to $5 \mathrm{~m}$, and deploy an unknown signal source $60 \mathrm{~m}$ away from the sensor network in advance. It can be seen that the size of the sensor coverage area depends not only on the number of sensors but also on the sensing range of the sensor itself. In addition, due to the limited energy of the sensor, the sensing range is very limited.

Figures 7 and 8 are the plane and three-dimensional schematic diagrams of the signal receiving intensity of the sensor network, respectively. Since the sensor network lacks prior information for non-cooperative signals, it can only be detected and judged by the strength of the received signal. For the convenience of presentation, the signal receiving strength in the figure is not converted into $\mathrm{db}$ form $(-70 \mathrm{dbm} \sim-38 \mathrm{dbm})$. It can be seen from Figure 7 that in a sensor network, when a beam signal is detected, the signal receiving strength of the sensor in a certain area will increase in detail, and the signal strength will gradually decrease with fading. The three-dimensional histogram in Figure 8 clearly shows the characteristics of the received signal strength of the sensor network when the directional beam signal is fading. On the other hand, it can be seen that due to the influence of sensor noise, it is very likely to affect the global detection probability.

Figure 9 shows the detection performance of the energy detection under different SNR $(-20 \mathrm{~dB} \sim 5 \mathrm{~dB})$ and the number of samples $N_{s}(64,512,2048)$ at $i$-th sensor. From Figure 9, we can obtain that the detection performance of $i$ th sensor increases with the increase of the SNR. The reason is that when the SNR increases, the signal strength of the beam also increases, and the noise is relatively small, so the sensor can better distinguish the signal of the beam, and the detection probability also increases. It also can be seen that

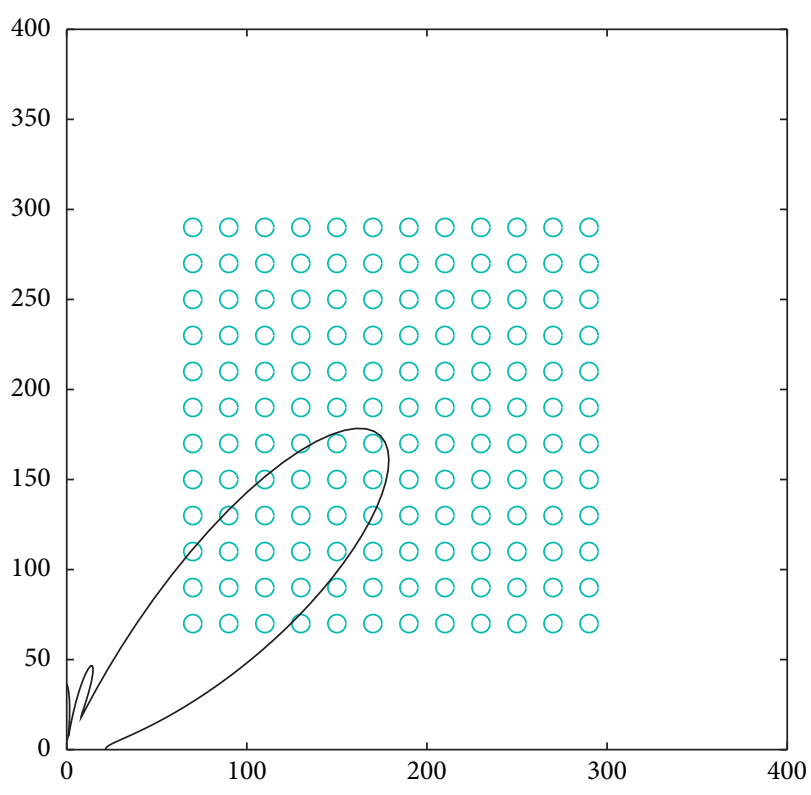

FIGURE 6: Schematic diagram of non-cooperative beam in $45^{\circ}$.

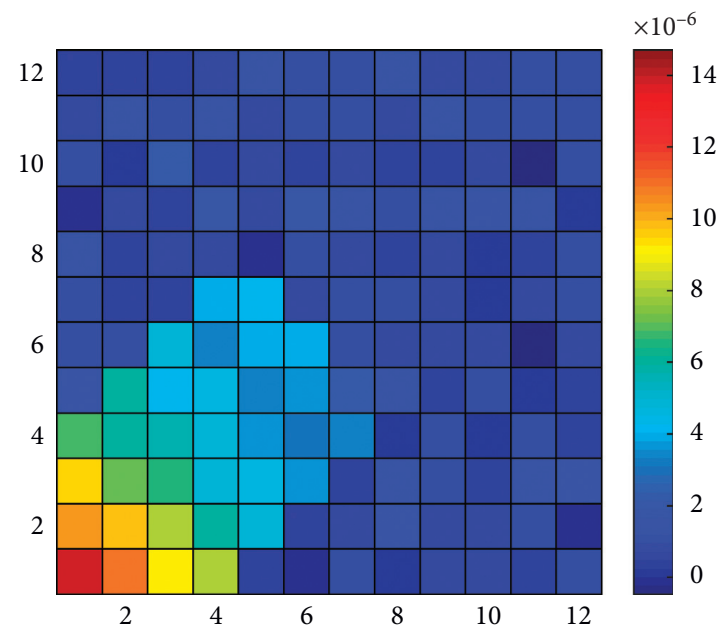

FIGURE 7: Schematic diagram of sensor network sensing beam signal strength (2D).

when $N_{s}$ increases, the detection performance of the $i$-th sensor will be enhanced, and it can still maintain a high detection performance under a low $\operatorname{SNR}(-10 \mathrm{~dB} \sim-5 \mathrm{~dB})$ ). When the SNR is constant, the more the sampling points of the sensor, the higher the detection probability. This is because increasing the number of sampling points can increase the observation data of the detection signal and the increase in the observation data can make the sensor's judgment more accurate and improve the detection probability. The simulation result accords with the formula (8). In the actual environment, due to channel fading, sensors closer to the beam radiation source will have better channel conditions. Therefore, we can improve the detection performance of $i$-th sensor by improving channel quality and increasing the number of samples. 


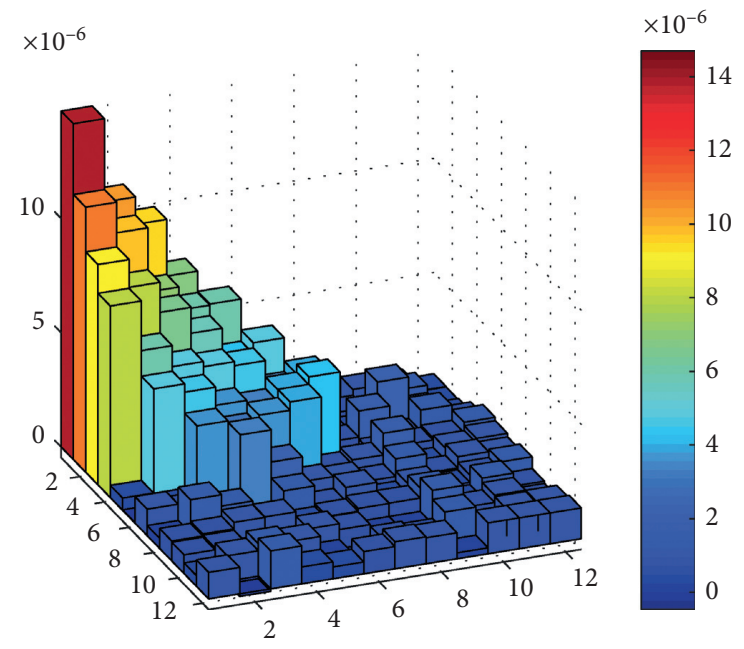

FIGURE 8: Schematic diagram of sensor network sensing beam signal strength (3D).

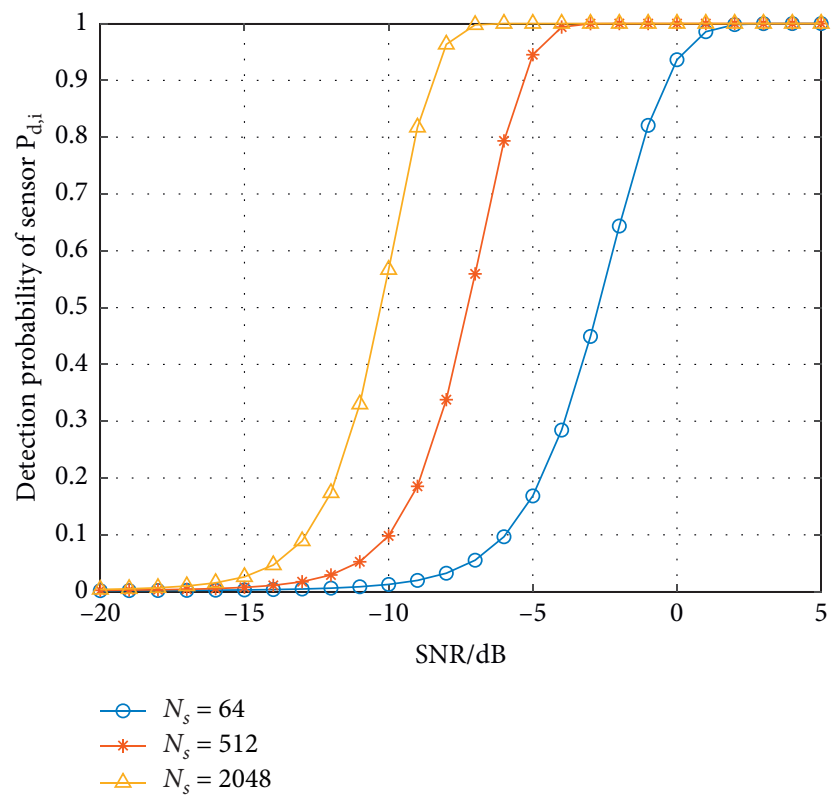

FIGURE 9: Energy detection performance under different SNR and $N_{s}$.

Figure 10 shows the detection performance of the energy detection under different $P_{f}$. As the false alarm probability increases, the detection probability below $-5 \mathrm{~dB}$ will increase. According to the formula (7), when the false alarm probability increases, the decision threshold is reduced, while detection probability will increase at this time. The simulation results also verify the correctness of the theoretical derivation.

Figure 11 plots the effect of different fusion algorithms on detection performance. It describes the change in detection performance when the detection probability of single sensor increases with different values of $k$. According to Algorithm 1, the simulation result is $N_{\text {opt }}=64$ when $N=144$. It can be seen that the performance of "AND" fusion rule and "OR" rule algorithms are the two extremes of the fusion algorithm. The overall detection probability increases when the single sensor detection probability is higher. The detection performance of our improved algorithm is improved over Schwartz algorithm by selecting $N_{\text {opt }}$. In half-voting algorithm, when the detection probability of a single sensor is greater than 0.5 , the detection performance of the proposed algorithm is better.

In order to integrate the detection probability and false alarm probability and to better evaluate the detection performance of the network, Figure 12 illustrates error probability at different $k$ values under formula (10) when $p\left(H_{0}\right)=p\left(H_{1}\right)=1 / 2$. It can be noted that the proposed algorithm has a lower error probability. In the half-voting 


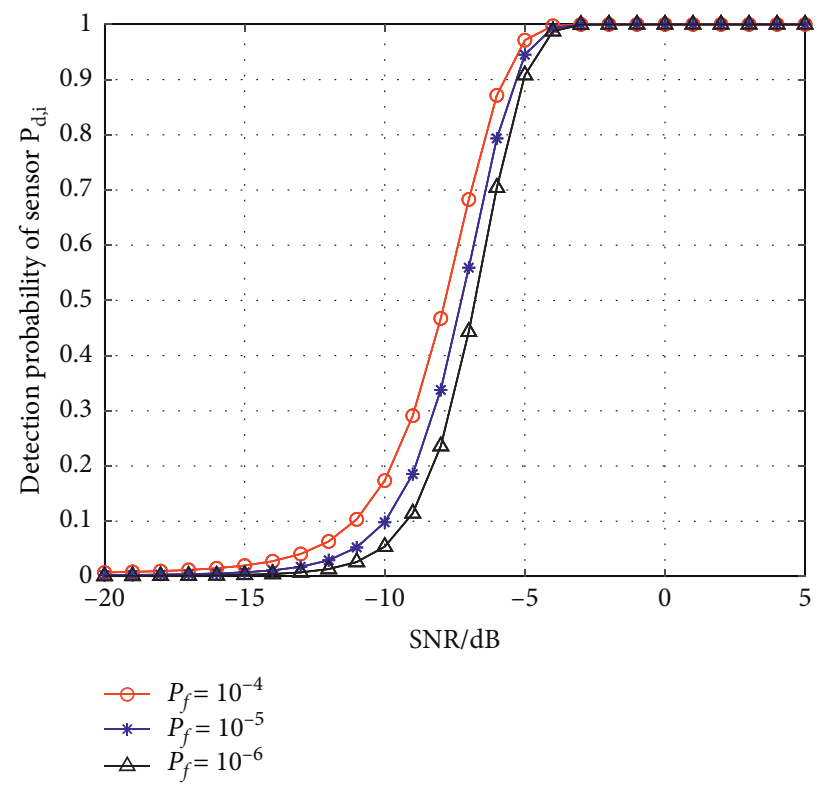

Figure 10: Energy detection performance under different SNR and $P_{f}$.

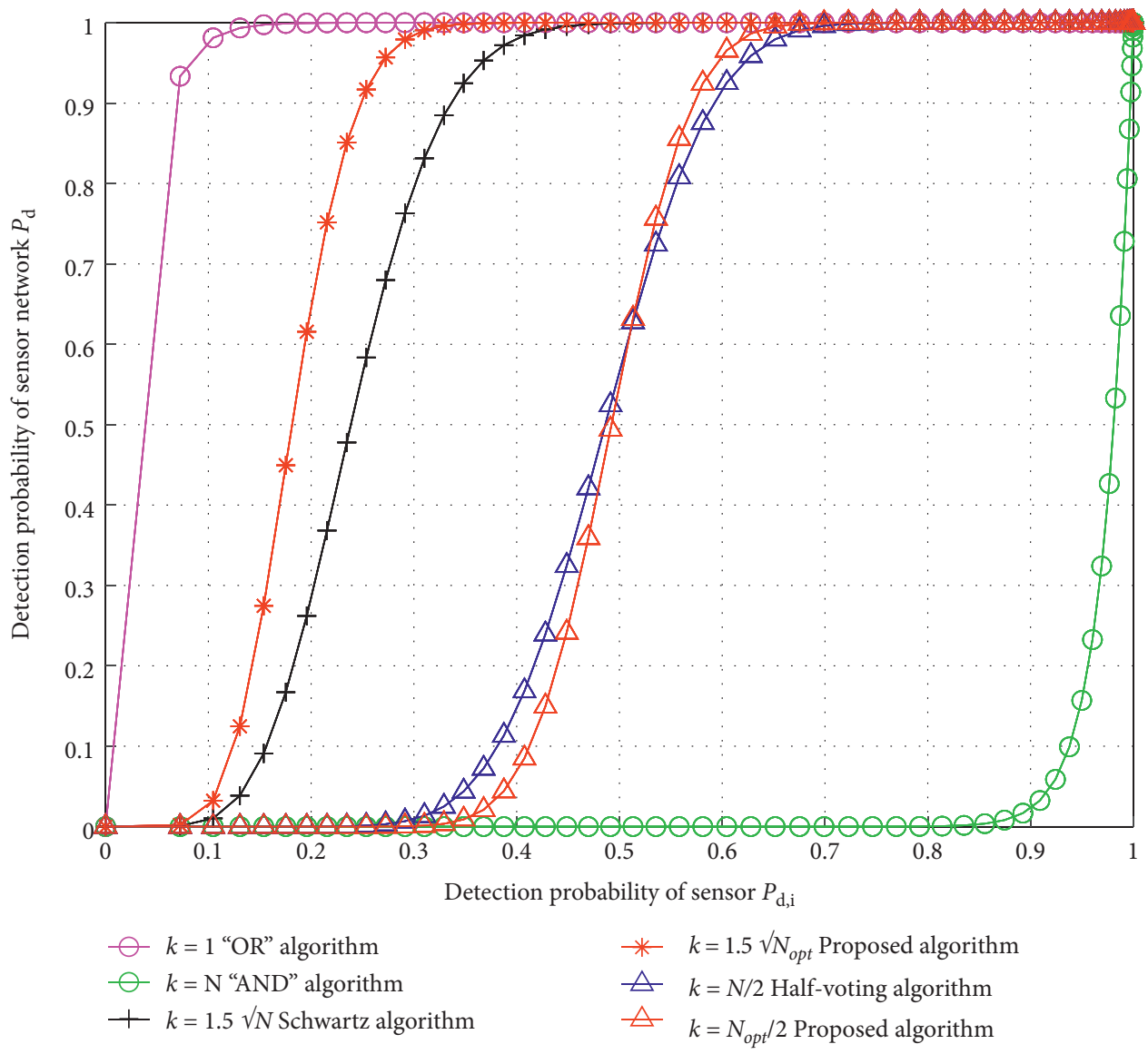

FIGURE 11: Detection probability comparison of different algorithms. 


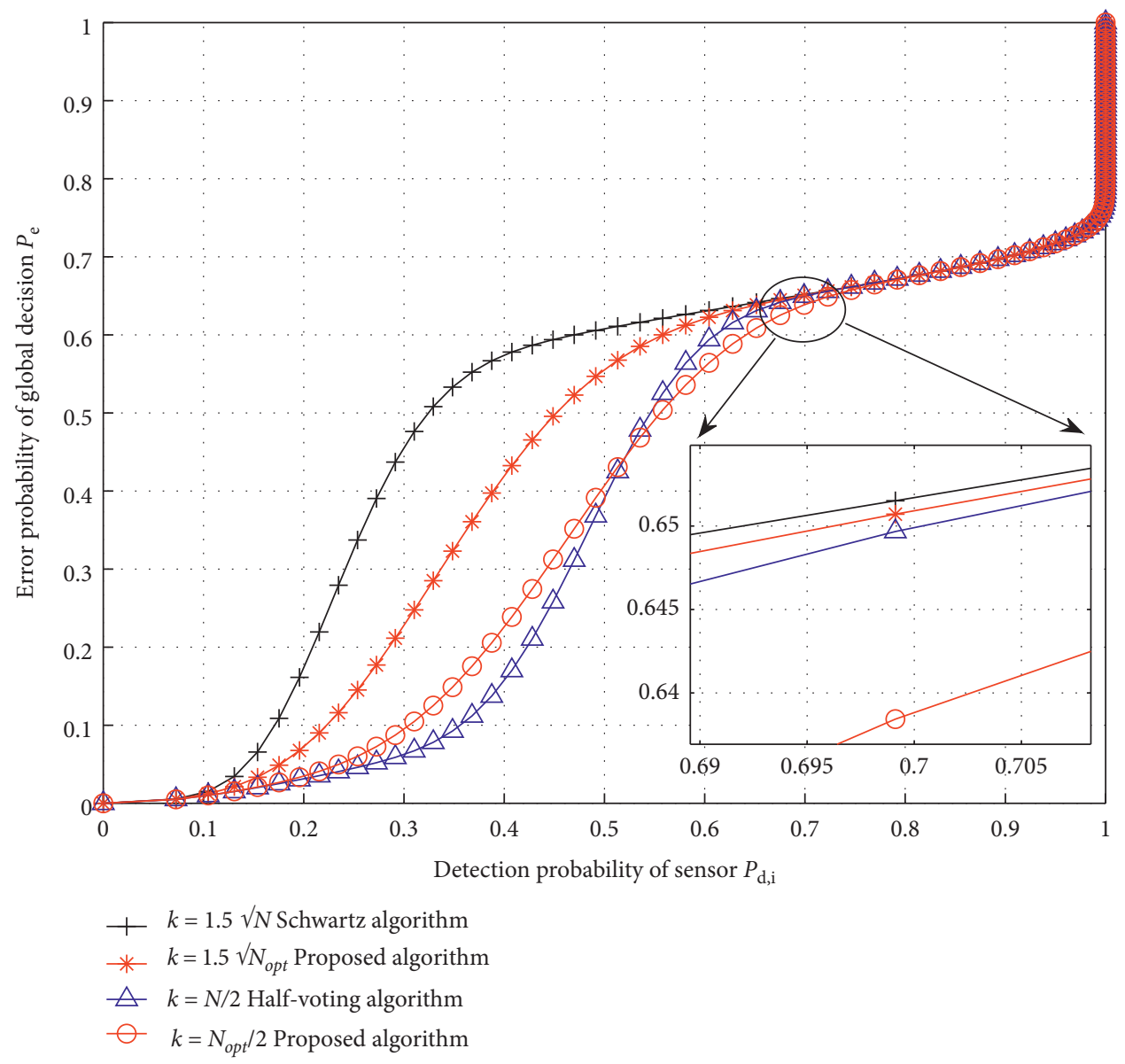

FIGURE 12: Error probability comparison of different algorithms.

algorithm, when the detection probability is less than 0.5 , the error probability of the proposed algorithm is higher. Because according to Figure 11, the detection probability of the proposed algorithm is relatively low at this time. When the detection probability is greater than 0.5 , the error probability of proposed algorithm is significantly lower than the halfvoting algorithm. Because in our algorithm, a large number of low detection probability sensors that are not covered by the beam are reduced, making the decision-making of the entire network more reasonable.

\section{Conclusions}

In this paper, we mainly constructed a beam signal sensing scheme based on wireless sensor networks. According to the characteristics of beam information, First, we derived the detection probability expression under the given false alarm probability. Second, we improved the " $k$ rank" fusion rule and selected $N_{\text {opt }}$ based on the position information of the sensor. Finally, we provided some simulation results and gained the effectiveness of the algorithm. Through simulation analysis, we found some interesting findings in the energy detection, such as by improving the SNR and increasing the number of samples will improve the overall detection performance. Through comparison with different fusion algorithms, our proposed algorithm showed better detection probability and lower error probability.
Abbreviations
BF:
Beamforming
WSNs: Wireless sensor networks
IoT: Internet of Things
NOMA: Nonorthogonal multiple access
D2D: Device-to-device networks
mmWave: Millimeter wave
RSS: $\quad$ Received signal strength
6G: The sixth generation
5G: $\quad$ The fifth generation
QAM: Quadrature amplitude modulation
MIMO: Multiple-input multiple-output
FC: $\quad$ Fusion center
AWGN: Additive white Gaussian noise
ED: $\quad$ Energy detection
SNR: $\quad$ Signal to noise ratio.

\section{Data Availability}

No data were used to support this study. 


\section{Conflicts of Interest}

The authors declare that they have no conflicts of interest regarding the publication of this article.

\section{Acknowledgments}

This work was supported by the National Key R\&D Program of China under Grant 2018YFB1801103, the National Natural Science Foundation of China (nos. 61871398, 61931011, and 62001517), the Natural Science Foundation for Distinguished Young Scholars of Jiangsu Province (no. BK20190030), and the Equipment Advanced Research Field Foundation (no. 61403120304).

\section{References}

[1] W. Saad, M. Bennis, and M. Chen, "A vision of $6 \mathrm{G}$ wireless systems: applications, trends, technologies, and open research problems," IEEE Network, vol. 34, no. 3, pp. 134-142, 2020.

[2] W. Roh, J.-Y. Seol, J. Park et al., "Millimeter-wave beamforming as an enabling technology for $5 \mathrm{G}$ cellular communications: theoretical feasibility and prototype results," IEEE Communications Magazine, vol. 52, no. 2, pp. 106-113, 2014.

[3] X. Li, H. Mengyan, Y. Liu, V. G. Menon, A. Paul, and Z. Ding, "I/Q imbalance aware nonlinear wireless-powered relaying of B5G networks: security and reliability analysis," IEEE Transactions on Network Science and Engineering, 1 page, 2020.

[4] B. Li, Z. Fei, C. Zhou, and Y. Zhang, "Physical-layer security in space information networks: a survey," IEEE Internet of Things Journal, vol. 7, no. 1, pp. 33-52, 2020.

[5] A. F. Molisch, V. V. Ratnam, S. Han et al., "Hybrid beamforming for massive MIMO: a survey," IEEE Communications Magazine, vol. 55, no. 9, pp. 134-141, 2017.

[6] S. A. Busari, K. M. S. Huq, S. Mumtaz, L. Dai, and J. Rodriguez, "Millimeter-wave massive MIMO communication for future wireless systems: a survey," IEEE Communications Surveys \& Tutorials, vol. 20, no. 2, pp. 836-869, 2018.

[7] S. Rangan, T. S. Rappaport, and E. Erkip, "Millimeter-wave cellular wireless networks: potentials and challenges," Proceedings of the IEEE, vol. 102, no. 3, pp. 366-385, 2014.

[8] M. S. Khan, S. J. Maeng, and Y. S. Cho, "Cell selection technique for Millimeter-Wave cellular systems with hybrid beamforming," Sensors, vol. 17, no. 6, pp. 1424-8220, 2017, https://www.mdpi.com/1424-8220/17/6/1461.

[9] A. Puglielli, A. Townley, G. LaCaille et al., "Design of energyand cost-efficient massive MIMO arrays," Proceedings of the IEEE, vol. 104, no. 3, pp. 586-606, 2016.

[10] Q. Qi, X. Chen, and D. W. K. Ng, "Robust beamforming for NOMA-based cellular massive IoT with SWIPT," IEEE Transactions on Signal Processing, vol. 68, pp. 211-224, 2020.

[11] X. Li, M. Zhao, Y. Liu, L. Li, Z. Ding, and A. Nallanathan, "Secrecy analysis of ambient backscatter NOMA systems under I/Q imbalance," IEEE Transactions on Vehicular Technology, p. 1, 2020.

[12] Z. Lin, M. Lin, Y. Huang, T. d. Cola, and W.-P. Zhu, "Robust multi-objective beamforming for integrated satellite and high altitude platform network with imperfect channel state information," IEEE Transactions on Signal Processing, vol. 67, no. 24, pp. 6384-6396, 2019.

[13] J. Choi, V. Va, N. Gonzalez-Prelcic, R. Daniels, C. R. Bhat, and R. W. Heath, "Millimeter-wave vehicular communication to support massive automotive sensing," IEEE Communications Magazine, vol. 54, no. 12, pp. 160-167, 2016.

[14] N. Deng, M. Haenggi, and Y. Sun, "Millimeter-wave deviceto-device networks with heterogeneous antenna arrays," IEEE Transactions on Communications, vol. 66, no. 9, pp. 42714285, 2018.

[15] N. Deng, Y. Sun, and M. Haenggi, "Success probability of millimeter-wave D2D networks with heterogeneous antenna arrays," in Proceedings of the 2018 IEEE Wireless Communications and Networking Conference, pp. 1-5, Las Vegas, Nevada, USA, 2018.

[16] B. Yang, Z. Yu, J. Lan, R. Zhang, J. Zhou, and W. Hong, "Digital beamforming-based massive MIMO transceiver for $5 \mathrm{G}$ millimeter-wave communications," IEEE Transactions on Microwave Theory and Techniques, vol. 66, no. 7, pp. 34033418, 2018.

[17] F. C. Robey, S. Coutts, D. Weikle, J. C. McHarg, and K. Cuomo, "MIMO radar theory and experimental results," in conference record of the thirty-eighth asilomar conference on signals Systems and Computers, pp. 300-304, Pacific Grove, CA, USA, 2004.

[18] J. Xu, L. Liu, and R. Zhang, "Multiuser MISO beamforming for simultaneous wireless information and power transfer," IEEE Transactions on Signal Processing, vol. 62, no. 18, pp. 4798-4810, 2014.

[19] A. Merwaday, R. Vannithamby, M. M. Rashid, Yi Zhang, C. Chen, and $\mathrm{X} . \mathrm{Wu}$, "On the performance of directional communications in ultra-dense networks," in Proceedings of the EEE International Conference on Communications Workshops (ICC Workshops), pp. 522-527, Shanghai, China, 2017.

[20] V. Raghavan, S. Subramanian, J. Cezanne, and A. Sampath, "Directional beamforming for millimeter-wave MIMO systems," in Proceedings of the 2015 IEEE Global Communications Conference (GLOBECOM), pp. 1-7, San Diego, CA, USA, 2015.

[21] B. Li, Z. Fei, Y. Zhang, and M. Guizani, "Secure UAV communication networks over 5G," IEEE Wireless Communications, vol. 26, no. 5, pp. 114-120, 2019.

[22] S. Abeywickrama, R. Zhang, Q. Wu, and C. Yuen, "Intelligent reflecting surface: practical phase shift model and beamforming optimization," IEEE Transactions on Communications, vol. 1-1, 2020 .

[23] T. Zheng, H. Wang, D. W. K. Ng, and J. Yuan, "Multi-antenna covert communications in random wireless networks," IEEE Transactions on Wireless Communications, vol. 18, no. 3, pp. 1974-1987, 2019.

[24] G. Ding, Q. Wu, F. Song, and J. Wang, "Spectrum sensing in opportunity-heterogeneous cognitive radio networks: noncooperative case," in Proceedings of the 2011 International Conference on Wireless Communications and Signal Processing (WCSP), pp. 1-5, Nanjing, China, 2011.

[25] S. Haghighatshoar and G. Caire, "The Beam alignment problem in mmWave wireless networks," in Proceedings of the 2016 50th Asilomar Conference on Signals, Systems and Computers, pp. 741-745, Pacific Grove, CA, USA, November 2016.

[26] G. Barriac, R. Mudumbai, and U. Madhow, "Distributed Beamforming for Information Transfer in Sensor Networks," in Proceedings of the Third International Symposium on Information Processing in Sensor Networks, pp. 81-88, New York, NY, April 2004.

[27] P. Zhang, I. Nevat, G. W. Peters, G. Xiao, and H. Tan, "Event detection in wireless sensor networks in random spatial 
sensors deployments," IEEE Transactions on Signal Processing, vol. 63, no. 22, pp. 6122-6135, 2015.

[28] L. Zhang, H. Zhao, S. Hou et al., "A survey on 5G millimeter wave communications for UAV-assisted wireless networks," IEEE Access, vol. 7, pp. 117460-117504, 2019.

[29] R. Zhang, H. Zhang, and W. Xu, "Fast beam alignment algorithm for multi-user mmWave communications," Electronics Letters, vol. 54, no. 25, pp. 1456-1458, 2018.

[30] Q. Xu, C. Jiang, Y. Han, B. Wang, and K. J. R. Liu, "Waveforming: an overview with beamforming," IEEE Communications Surveys \& Tutorials, vol. 20, no. 1, pp. 132-149, 2018.

[31] Z. Lin, M. Lin, J. Ouyang, W. Zhu, and S. Chatzinotas, "Beamforming for secure wireless information and power ransfer in terrestrial networks coexisting with satellite networks," IEEE Signal Processing Letters, vol. 25, no. 8, pp. 166-1170, 2018.

[32] H. Liu, J. Chen, G. Ding, T. A. Tsiftsis, and C. Rowell, "Antenna beamforming for energy harvesting in cognitive radio etworks," in Proceedings of the 2016 IEEE MTT-S International Wireless Symposium (IWS), pp. 1-4, Shanghai, China, 2016.

[33] M. Z. Hasan and H. Al-Rizzo, "Beamforming optimization in internet of things applications using robust swarm lgorithm in conjunction with connectable and collaborative sensors," Sensors, vol. 20, no. 7, 2020.

[34] X. Yu, J. Zhang, M. Haenggi, and K. B. Letaief, "Coverage analysis for millimeter wave networks: the impact of directional antenna arrays," IEEE Journal on Selected Areas in Communications, vol. 35, no. 7, pp. 1498-1512, 2017.

[35] X. Zhang, H. V. Poor, and M. Chiang, "Optimal power allocation for distributed detection over MIMO channels in wireless sensor networks," IEEE Transactions on Signal Processing, vol. 56, no. 9, pp. 4124-4140, 2008.

[36] P. Zhang, J. Y. Koh, S. Lin, and I. Nevat, "Distributed event detection under byzantine attack in wireless sensor etworks," in Proceedings of the IEEE Ninth International Conference on Intelligent Sensors, Sensor Networks and Information Processing (ISSNIP), Singapore, pp. 1-6, April 2014.

[37] D. Ciuonzo, G. Romano, and P. Salvo Rossi, "Performance analysis and design of maximum ratio combining in hannelaware MIMO decision fusion," IEEE Transactions on Wireless Communications, vol. 12, no. 9, pp. 4716-4728, 013.

[38] I. Nevat, G. W. Peters, and I. B. Collings, "Distributed detection in sensor networks over fading channels with multiple antennas at the fusion centre," IEEE Transactions on Signal Processing, vol. 62, no. 3, pp. 671-683, 2014.

[39] M. Tavana, A. Rahmati, V. Shah-Mansouri, and B. Maham, "Cooperative sensing with joint energy and correlation detection in cognitive radio networks," IEEE Communications Letters, vol. 21, no. 1, pp. 132-135, 2017.

[40] S. Atapattu, C. Tellambura, and H. Jiang, "Energy detection based cooperative spectrum sensing in cognitive radio networks," IEEE Transactions on Wireless Communications, vol. 10, no. 4, pp. 1232-1241, 2011.

[41] Y. Liang, Y. Zeng, E. C. Y. Peh, and A. T. Hoang, "Sensingthroughput tradeoff for cognitive radio networks," IEEE Ransactions on Wireless Communications, vol. 7, no. 4, pp. 1326-1337, 2008.

[42] H. Urkowitz, "Energy detection of unknown deterministic signals," Proceedings of the IEEE, vol. 55, no. 4, pp. 23-531, 1967.

[43] F. F. Digham, M. . Alouini, and M. K. Simon, "On the energy detection of unknown signals over fading channels," $E E E$
International Conference on Communications, vol. 5, pp. 3575-3579, 2003.

[44] M. Schwartz, "A coincidence procedure for signal detection," IEEE Transactions on Information Theory, vol. 2, no. 4, pp. 135-139, 1956.

[45] M. R. Akdeniz, Y. Liu, M. K. Samimi et al., "Millimeter wave channel odeling and cellular capacity evaluation," IEEE Journal on Selected Areas in Communications, vol. 32, no. 6, pp. 164-1179, 2014. 